\title{
Automated Tracking of Multiple C. Elegans
}

\author{
Ebraheem Fontaine*, Joel Burdick*, Alan Barr ${ }^{\dagger}$ \\ ${ }^{*}$ Department of Mechanical Engineering, California Institute of Technology, Pasadena, CA 91125 \\ Email: \{ebraheem,jwb\}@ robotics.caltech.edu \\ $\dagger$ Department of Computer Science California Institute of Technology, Pasadena, CA 91125 \\ Email: barr@gg.caltech.edu
}

\begin{abstract}
This paper presents a method for model based automated tracking of multiple worm-like creatures. These methods are essential for accurate quantitative analysis into the genetic basis of behavior that involve more than one organism. An accurate worm model is designed using the geometry of planar curves and nonlinear estimation of the model's parameters are performed using a central difference Kalman filter (CDKF). The filter can naturally be expanded to estimate the locations of multiple worms and determine when they are occluding each other. The predicted location of the models at each iteration allows for an efficient method to determine the regions that are undergoing occlusions. Experiments on actual C. Elegans mating sequence data demonstrate the quality of the proposed method.
\end{abstract}

\section{INTRODUCTION}

The small nematode Caenorhabditis elegans (C. Elegans) is a widely used model organism in the study of genetics and developmental biology. Since the exact position and cell lineage of all of its 959 cells and 302 neurons is known, C. Elegans offers a convenient platform to understand how different behaviors are modulated by the nervous system, sensory input, and genetic modifications. However, to study the relationship between behavior and genes in C. Elegans, for example, requires the screening of an enormous number of individual $C$. Elegans specimens to establish the genebehavior relationship. Each screen consists of a visual observation of the specimen's movements over a time period, and the extraction of key movement and behavior parameters from the observations. The number and complexity of the observations that are needed to support current research into the gene-behavior linkage argues for highly automated screening systems.

Several automated tracking and analysis systems have been previously developed for $C$. Elegans. These tracking systems can be roughly divided into two categories. The first class of systems track multiple worms at a low magnification, and they are able to capture the animals' gross motion characteristics, such as velocity and frequency of reversals [1]. Systems in the second category track single worms at a higher magnification and utilize a motorized stage to keep the worm in the camera field of view [2], [3]. These systems can quantify the detailed posture of the individual worms, and subsequently perform phenotype classification. However, limitations in the underlying implementations of these systems prevent them from tracking detailed worm posture for multiple organisms that may occlude each other.
Specifically, mating behavior is a key biological process which involves two worms that severely occlude each other through long periods of time. To understand how genes control the neuronal and sensory function of the worms during mating, the detailed kinematics of the organisms must be captured during interactions.

This paper presents new methods for automated tracking of multiple $C$. Elegans specimens during complex behavior, such as mating, which involves significant occlusion. Our tracking algorithm combines detailed geometric models with nonlinear estimation techniques to quantify the shape and motion of the worms. We demonstrate this algorithm on two video sequences, and automatically extract key kinematic parameters. The paper is organized as follows. Section II describes the modeling approach, while Section III summarizes the main tracking algorithm. Sections V and VI discuss the results and conclusions.

\section{GEOMETRIC MODELING}

The motion of $C$. Elegans on a typical microscope slide is largely planar, so we restrict our modeling of the worm to two dimensions. Three dimensional effects can be relatively easily added if desired. To a good approximation, C. Elegans maintains a constant length and width during locomotion. Although a worm may undergo elongations and contractions during mating behavior, the magnitude of these effects is small enough to ignore. Therefore, we model the centerline of each worm as a planar, inextensible curve. The body posture can be effectively described by the bend angle of the worm as a function of distance along its length. For practicality, this function, $\Theta(u)$, is finitely parameterized using a linear combination of known basis functions, $\Phi_{j}^{k}(u)$ : $\Theta(u)=\sum_{j=1}^{N_{\Theta}} \alpha_{j} \Phi_{j}^{k}(u)$. The current implementation uses a fourth order $(k=4)$ periodic B-spline basis with $N_{\Theta}=8$, though the results are not highly sensitive to these choices. The tangent and normal vectors of the centerline are defined as

$$
e_{1}=\left[\begin{array}{c}
\cos (\Theta(u)) \\
\sin (\Theta(u))
\end{array}\right] \quad e_{2}=\left[\begin{array}{c}
-\sin (\Theta(u)) \\
\cos (\Theta(u))
\end{array}\right]
$$

To account for the worm's finite width, our model is a two dimensional surface defined around the centerline curve:

$$
\begin{gathered}
F(u, v)=\beta\left(\int_{0}^{u} e_{1}(\hat{u}) d \hat{u}+v R(u) e_{2}(u)\right)+\vec{T} \\
u \in\left[-\frac{L}{2}, \frac{L}{2}\right] \quad v \in[-1,1]
\end{gathered}
$$


where $\beta$ is a constant scaling term with units $\frac{\text { pixels }}{m m}, \vec{T}$ is a global translation vector to the model's center of mass, $L$ is the worm's length, and $R(u)$ is a continuous function for the worm's radius as a function of distance along its length. Currently $R(u)$ is defined as a fourth order non-periodic B-spline using eight control points. Since the model is evaluated on a fixed $(u, v)$ mesh during tracking, it becomes parameterized by $\alpha_{j}$ and $\vec{T}$. Fig. 1 shows the worm model in a bent configuration. For our experiments, the constant scaling term $\beta$ is directly calculated as $340 \frac{\text { pixels }}{\mathrm{mm}}$ using a stage micrometer.

Our approach to modeling C. Elegans offers several advantages. Since each B-spline basis function is only defined over a subset of the domain, the control points, $\alpha_{j}$, have local control over the shape. This property is analogous to the worm's anatomy, where groups of muscles contract over local body regions. In addition, our parametrization of the centerline offers a natural means to constrain the worm's length. Our model can allow for elongations and contractions via a rate of length function, $K(u): \vec{x}(u)=$ $\int_{0}^{u} K(\hat{u}) e_{1}(\Theta(\hat{u})) d \hat{u}$.

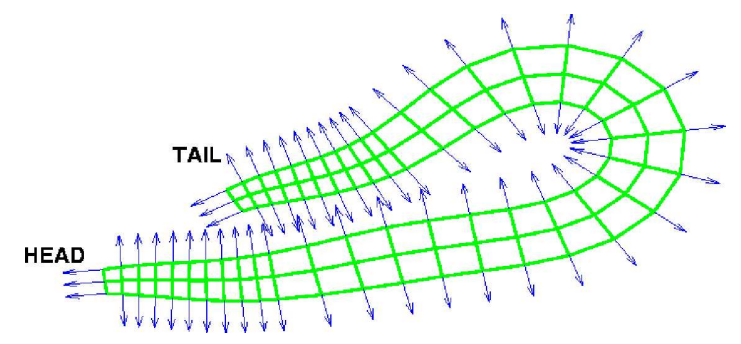

Fig. 1. Worm Model in a bent configuration with outward normal vectors assigned along the boundary.

\section{NONLINEAR FILTERING}

Visual tracking problems can be formulated as the estimation of a state, $p$, via measurements, $z$, in a discrete time dynamic state space model:

$$
\begin{aligned}
p_{k} & =f\left(p_{k-1}, \xi_{k-1}\right) \\
z_{k} & =h\left(p_{k}, v_{k}\right)
\end{aligned}
$$

where $\xi_{k}$ and $v_{k}$ are noise processes, which we assume are white and Gaussian.

Several popular nonlinear estimation techniques, such as extended Kalman filters (EKF) [4], Sigma Point Kalman filters (SPKF) [5], and particle filters [6] could be used for this problem. We use one of the SPKFs known as a central-difference Kalman Filter (CDKF) [7], [8]. This filter, which is based on Stirling's interpolation formula, has a comparatively accurate covariance estimate and is also parameterized by a single scalar which has an optimal value for Gaussian noise distributions [7]. For the sake of brevity, the filter equations are not repeated here, but can be found in [7], [8]. Sections III-A and III-B describe the filter equations for a single worm. A filter to track $K$ worms is achieved by concatenating the state and observation vectors $K$ times.

\section{A. Motion Model}

The motion model assumes that the worm moves with a constant wave velocity, $\eta_{1}$, corrupted by acceleration noise, $\eta_{2}$. Thus, the state vector and process noise vector for a single worm are:

$$
p=\left[\begin{array}{lll}
\vec{\alpha} & \vec{T} & \eta_{1}
\end{array}\right]^{T} \quad \xi=\left[\begin{array}{lll}
\Delta \vec{\alpha} & \Delta \vec{T} & \eta_{2}
\end{array}\right]^{T}
$$

The equations of motion calculate the predicted state vector after the worm has undergone a total wave displacement of $\eta_{0}=\eta_{1} \Delta t+\eta_{2} \frac{\Delta t^{2}}{2}$, where $\Delta t$ is inverse of the camera frame rate. These equations take the form:

$$
\begin{aligned}
p_{k}= & f\left(p_{k-1}, \xi_{k-1}\right) \\
{\left[\begin{array}{c}
\vec{\alpha}_{k} \\
T_{x_{k}} \\
T_{y_{k}} \\
\eta_{1_{k}}
\end{array}\right]=} & {\left[\begin{array}{c}
\Phi(u)^{-1} \Phi_{*}\left(u+\eta_{0}\right) \Phi_{*}(u)^{-1} \Phi(u) \vec{\alpha}_{k-1} \\
T_{x_{k-1}}+\int_{0}^{\eta_{0}} \cos \left(\Phi(\hat{u}) \vec{\alpha}_{k-1}\right) d \hat{u} \\
T_{y_{k-1}}+\int_{0}^{\eta_{0}} \sin \left(\Phi(\hat{u}) \vec{\alpha}_{k-1}\right) d \hat{u} \\
\eta_{1_{k-1}}
\end{array}\right] } \\
& +\left[\begin{array}{c}
\Delta \vec{\alpha}_{k-1} \\
\Delta T_{x_{k-1}} \\
\Delta T_{y_{k-1}} \\
\eta_{2_{k-1} \Delta t}
\end{array}\right]
\end{aligned}
$$

Here, $\Phi(u)$ is a $s \times N_{\Theta}$ matrix of B-spline bases evaluated at $s$ sampled grid points in $u$. B-spline functions are also defined by a knot vector, $\vec{\omega}$, with $\omega_{n} \leq \omega_{n+1}$, that assigns the domain over which the B-spline basis is defined. Here $\Phi(u)$ is defined only in the domain $u \in\left[-\frac{L}{2}, \frac{L}{2}\right]$, so the range of $u$ and $\vec{\omega}$ are identical. Defining the basis over the smallest domain possible increases the sensitivity of the shape parameters, $\vec{\alpha}$. However, this definition does not allow us to directly calculate $\vec{\alpha}_{k}$ in (5). Instead, we must define a new matrix of B-splines, $\Phi_{*}(u)$, which has a knot vector $\vec{\omega}_{*}$ defined over the domain $\left[-\frac{L}{2}-\eta_{0}, \frac{L}{2}+\eta_{0}\right]$ to account for the worm's displacement. The predicted shape parameters are then calculated by projecting onto this basis, calculating the new $\Theta(u)$, and projecting back onto the original basis, $\Phi(u)$.

\section{B. Observation Model}

The worm observation model is illustrated in Fig. 1, and it consists of the boundary points along with their outward normal vectors. These boundary points can be divided into two categories: (1) head and tail points located at the ends of the model; and (2) dorsal and ventral points located along the model length. Letting $q_{i}=\left[\begin{array}{ll}x_{i} & y_{i}\end{array}\right]^{T}$ be the planar location of a boundary point, the observation vector is:

$$
\begin{aligned}
Q_{1} & =\left[\begin{array}{lll}
q_{\text {tail }_{1}} & \ldots & q_{\text {tail }_{N_{1}}}
\end{array}\right] \\
Q_{2} & =\left[\begin{array}{lll}
q_{\text {head }_{1}} & \ldots & q_{\text {head }_{N_{1}}}
\end{array}\right] \\
Q_{3} & =\left[\begin{array}{llll}
n_{\text {dorsal }_{1}}^{T} & q_{\text {dorsal }_{1}} & \ldots & n_{\text {dorsal }_{N_{2}}}^{T} q_{\text {dorsal }_{N_{2}}}
\end{array}\right] \\
Q_{4} & =\left[\begin{array}{llll}
n_{\text {ventral }_{1}}^{T} q_{\text {ventral }_{1}} & \ldots & n_{\text {ventral }_{N_{2}}}^{T} q_{\text {ventral }_{N_{2}}}
\end{array}\right] \\
z_{k} & =\left[\begin{array}{llll}
Q_{1} & Q_{2} & Q_{3} & Q_{4}
\end{array}\right]^{T}
\end{aligned}
$$

where $N_{1}$ and $N_{2}$ are the number of points in category 1 and 2 , respectively. These numbers are determined by the size of the $(u, v)$ mesh used. The observation vector 
includes the inner product of the dorsal and ventral points with their corresponding normal vector. When the actual measurements, $r_{i}$, of these points are observed, they are also projected onto $n_{i}$ so that the innovation vector has the form $\mathbf{n}^{\mathbf{T}}(\mathbf{q}-\mathbf{r})$.

\section{Feature Extraction And CorRespondence}

The raw worm images are segmented into foreground and background pixels using a simple thresholding technique. Because lighting often causes bright areas in the middle of the worm's silhouette, a hole-filling operation is used similar to that found in [3]. Boundaries in the binary image are then found using the built in MATLAB $R$ function 'bwboundaries'. A B-spline curve is then fitted to all boundaries above a certain length, and the outward normal vectors, $m_{j}$, and locations of high negative curvature, $\kappa_{j}$, are calculated. These high curvature feature points are important for localizing the worm since they represent head and tail locations. Fig. 2 illustrates the process on a typical image. The model

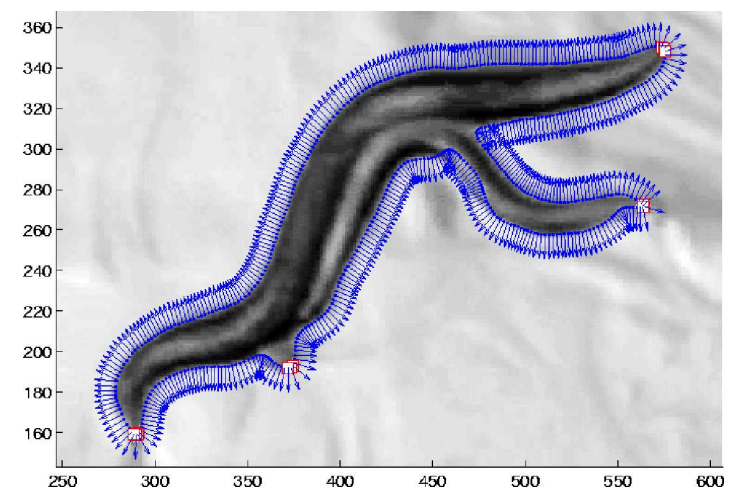

Fig. 2. Raw image of worms engaged in mating behavior with extracted features illustrated. Axis units are in pixels.

points, $q_{i}$ are matched with corresponding data points, $r_{j}$ by maximizing the following likelihood criteria:

$$
\Lambda_{i j}=\frac{1}{\sqrt{\operatorname{det}\left(2 \pi S_{i}\right)}} e^{-\frac{1}{2}\left(q_{i}-r_{j}\right)^{T} S_{i}^{-1}\left(q_{i}-r_{j}\right)}
$$

where $S_{i}$ is the $2 \times 2$ covariance matrix associated with $q_{i}$. In addition to the maximum likelihood criteria, we also require that the corresponding normal vectors have similar orientation (their inner product is greater than a parameter $\rho$ ). The point $q_{i}$ is corresponded with point $r^{*}$ using:

$$
r^{*}=\left\{r \in\left\{r_{j}\right\}: \max _{j}\left\{\Lambda_{i j}\right\}, \quad n_{i}^{T} m_{j} \geq \rho\right\}
$$

For the model locations that correspond to the head and tail points, we use (8) with the set $\left\{r_{j}\right\}$ replaced with $\left\{\kappa_{j}\right\}$. Our data association methods allows for multiple assignments within a model to the same data point. However, different models cannot be assigned to the same data boundary point. This constraint arises from the fact that worms never share a visible boundary while they are occluding each other during mating.

Our algorithm handles occlusions using the matching criteria explained above. All model locations which do not find a corresponding data point are labeled as occluded. Occluded points reduce the dimension of $z_{k}$ and change the observation equation. At each filter iteration, we assume that the entire model is visible, and then determine which points are occluded. Since the number of occluded points may change at each frame, we iteratively define a new observation function, $h_{k}\left(p_{k}, v_{k}\right)$, that only returns the locations of visible boundary points.

\section{RESULTS}

We present the application of the tracking algorithm to two video sequences of $720 \times 480$ pixel gray-scale images which were 478 frames in length. In these video sequences, the hermaphrodite is partially paralyzed to facilitate tracking and to keep the behavior within the camera's field of view. The model parameters of each worm are manually initialized to match the configuration of the worms in the first video frame. Figs. 3 and 4 show the tracking results and videos can be viewed at http://robotics.caltech.edu/ ebraheem/Research/ biomotion.htm. To ensure high fidelity tracking, our algorithm monitors the current variance in the state estimate at each time step. If a state parameter variance grows beyond a specified threshold, the algorithm pauses, and allows the user to inspect the current estimate. If the estimate is accurate based on visual inspection, the user can restart the algorithm. If the estimate needs refining, the user can manually correct the model location. The rare tracking failures typically occur when both the head and tail of the worm remain occluded for more than a few frames.

The algorithm is written in MATLAB $\mathbb{R}$, utilizes parts of [9] in its implementation, and operates in a batch off-line mode. It currently tracks two worms at a rate of 2 frames per minute on a Pentium IV $3.0 \mathrm{GHz}$ machine. For the behavioral assays this algorithm is designed to support, accuracy, not speed, is the primary target. Future implementations in $\mathrm{C}++$ are likely to achieve much faster computation time.

Research into the genetic basis of mating behavior mainly involves studying the male motion. Fig. 5 shows kinematic parameters of a male wild-type worm estimated from the first video sequence. The velocity of the worm's motion and the time evolution of its shape are both important measurements in quantifying behavior.

\section{CONCLUSION}

This paper demonstrates a new algorithm for tracking multiple C. Elegans (or other worm-like creatures) during complex behavior. A detailed geometric model of the worm is combined with a Central Difference Kalman Filter to perform nonlinear estimation of the model parameters. The results show stable tracking of the worms even during occlusions. Future work will make the tracker more robust to extended occlusions observed during mating and facilitate the classification of various male phenotypes based on the extracted kinematic parameters. 


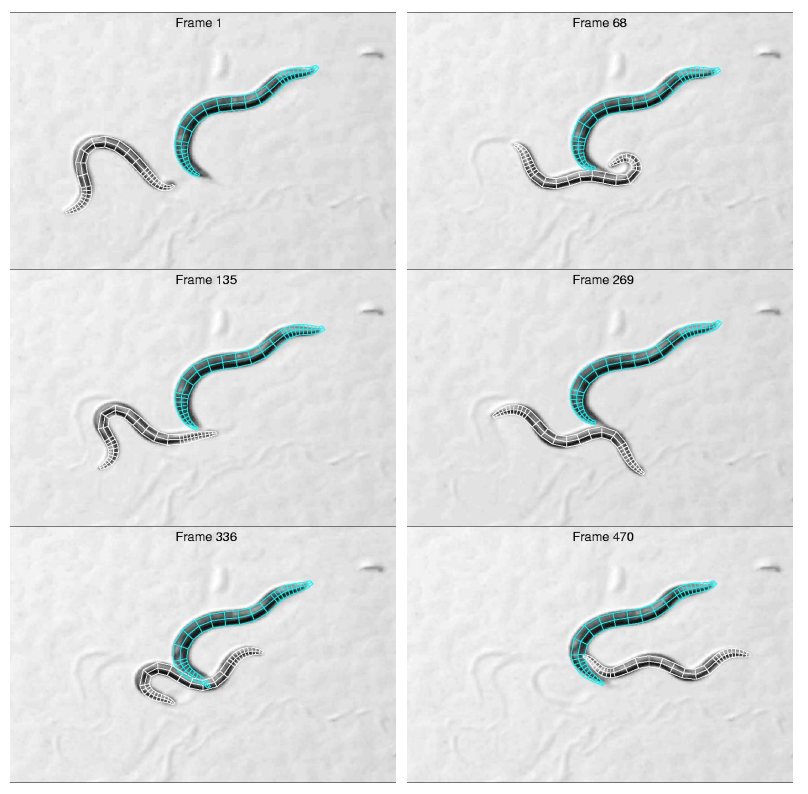

Fig. 3. Tracking sequence using the proposed tracker. See wormmating04demo1.mpg for details
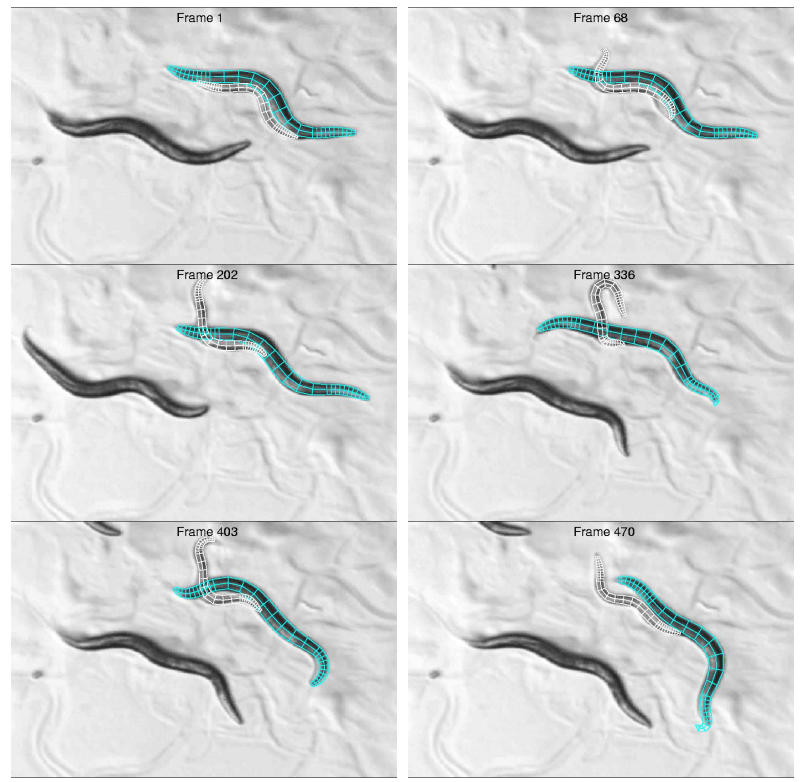

Fig. 4. Tracking sequence using the proposed tracker. See wormmating07demo1.mpg for details

\section{A. Acknowledgments}

The authors are grateful to the Beckman Institute Foundation for support of this work. We also thank Dr. Allyson Whittaker for supplying the video sequences used in this analysis.

\section{REFERENCES}

[1] M. de Bono and C. Bargmann, "Natural variation in a neuropeptide $\mathrm{y}$ receptor homolog modifies social behavior and food response in c. elegans," Cell, vol. 94, pp. 679-689, 1998.

[2] C. Cronin et al., "An automated system for measuring parameters of nematode sinusoidal movement," BMC Genetics, vol. 6, no. 1, Feb. 2005.

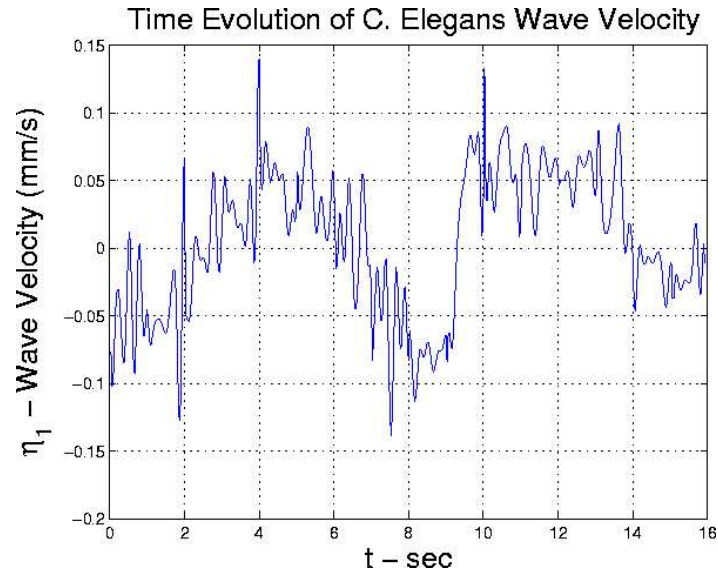

(a) Instantaneous wave velocity of the male worm during mating behavior

\section{Time Evolution of C. Elegans Body Posture}

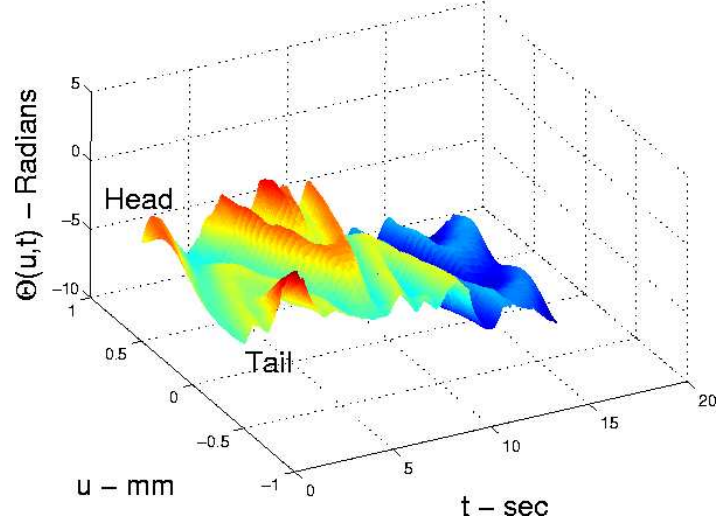

(b) Bend angle surface of the male worm during mating behavior

Fig. 5. Estimated kinematic parameters of the male worm during mating behavior. Data extracted from first tracking sequence

[3] W. Geng et al., "Automatic tracking, feature extraction and classification of c. elegans phenotypes," IEEE Trans. Biomed. Eng., vol. 51, no. 10, pp. 1811-1820, Oct. 2004.

[4] Y. Bar-Shalom, X. Li, and T. Kirubarajan, Estimation with Applications to Tracking and Navigation. John Wiley \& Sons, Inc., 2001.

[5] R. van der Merwe and E. Wan, "Sigma-point kalman filters for probabilistic inference in dynamic state-space models," in Proc. of the Workshop on Advances in Machine Learning, Montreal, Canada, June 2003.

[6] A. Doucet, N. de Freitas, and N. Gordon, Sequential Monte-Carlo Methods in Practice. Springer-Verlag, 2001.

[7] M. Nørgaard, N. Poulsen, and O. Ravn, "New developments in state estimation for nonlinear systems," Automatica, vol. 36, 2000.

[8] K. Ito and K. Xiong, "Gaussian filters for nonlinear filtering problems," IEEE Trans. Automat. Contr., vol. 45, no. 5, pp. 910-927, may 2000.

[9] R. van der Merwe and E. Wan, "Rebel:recursive bayesian estimation library," http://choosh.bme.ogi.edu/rebel/, 2004, matlab®Toolkit. 